\title{
Accident sites management using drones
}

\author{
Oliviu Mihnea Gamulescu ${ }^{1}$, Sebastian Daniel Rosca ${ }^{1 *}$, Fabian Panaite $^{1}$, Alin Costandoiu ${ }^{1}$, \\ and Simona Riurean ${ }^{1}$
}

${ }^{1}$ University of Petrosani, Department of Computer and Electrical Engineering, Universitatii str., no. 20, 332006, Petrosani, Romania

\begin{abstract}
Nowadays, finding a way to quickly respond to industrial accidents due to human errors or unforeseen situation is high, for example in the petrochemical industry where are handled with toxic and flammable substances, is a priority for the safe coordination of rescue teams based onreal time knowledge of the situation on the ground. Thus, the rapid identification of potential victims, the prevision of post-deflagration work space and the prevention of the new emergencies to limit damage should become a priority. In this paper we propose a quickly solution for rescue intervention based on use of the drone equipped with camera and sensors network capable of transmitting data in real time over the post-explosive environmental conditions to command center for the rapid identification of victims both in open and closed spaces. The paper presents the methods of using drones to explore and identify accident sites. There are modelled and simulated the control system and the image identification unit.
\end{abstract}

\section{Introduction}

Unmanned Aerial Vehicle technology has developed for the last 20 years. Lately, the size of drones has diminished, for both, the benefit of better access to isolated locations, and also reduced costs and low power consumption.

Disaster areas are often inaccessible, thus, most rescue operations, becoming incredibly difficult, requiring remote sensing technology to be used in real time, to gather information and provide efficient and effective rescue actions.

Disaster detection and response, had recently been using different sources of data, simultaneously, aiming to gather more detailed information about accidents, and to have central command make more effective, efficient, and reliable decisions in controlling the situation. UAV are now operable under deadly conditions and unreachable areas. In small sizes, better mobility and anytime access, drones can operate in a relatively harsh climate of the rescue or reconnaissance missions.

Stable network infrastructures are essential to the success of UAV search and rescue missions, making the presence of multiple connection types (802.11 Wi-Fi network, $2 \mathrm{G} / 3 \mathrm{G} / 4 \mathrm{G}$, and even UAV ad-hoc networks) a key provision.

*Corresponding author: sebastianrosca91@gmail.com 


\section{Problem description}

The causes of most industrial accidents are equally represented by natural calamities and human errors that can result from material damage to human life losses affecting a wide range of critical industries from the petrochemical industry to the transportation industry.

\subsection{Common accidents areas}

\subsubsection{Petrochemical industry}

Nowadays, it is difficult to imagine how our lifestyle could be without the contribution of 21 st century technology. Thus, the development of electronics from smartphones, PCs, and domestic appliances would not be possible without the input brought by the petrochemical industry. For example, without the chemical production of solvents, it would not be possible to produce solutions for soldering, scouring, degreasing of spare electronic parts, and other branches of industry such as biotechnology and precision farming could not be developed properly. Thus, the production of solvents and fuels used by agriculture machine and by the automotive industry is also the main cause of hazards of pollution due to inappropriate handling [1]. A study performed by researchers from Gonabad University of Medical Science from Iran has shown that $81 \%$ of chemicals used in the petrochemical industry can produce moderate to high-risk effects on employee health, so that up to one million workers in the chemical industry die annually or suffer from disabilities [2] due to inappropriate exposure to chemicals according to a statistics of the World Health Organization (WHO) [3].

Concerning the classification of explosion hazard situations in the petrochemical industry in descending order of danger, the most hazardous operations are related to refining and storage of fuels because of flammability: the gasoline present a major risk of deflagration and toxicity in the case of vapors at a limit of concentration between $1.3 \%$ and $6 \%$, hydrogen present a risk of deflagration at a limit of concentration between $4.1 \%$ and $74 \%$, and methane at a limit of concentration between $5.3 \%$ and $15 \%$ [4-5].

\subsubsection{Transportation Industry of dangerous substances}

The transportation of dangerous substances by water, land and air is regulated at European Union level by major accident hazards based on Directive 2012/18 / EU of the European Parliament and based on the Council Directive of 4 July 2012 [6]. According to world statistics, the transport of dangerous chemicals, estimated at 4 billion of tons annually, only on the road, poses a high risk of hazard because of the diversity of the chemical substances transported [7]. According to the United Nations, hazardous substances are transported into nine risk classes: explosive substances; gases; flammable liquids; flammable solids category they belong to: solid desensitized explosives and self-reactive substances; substances prone to spontaneous combustion, substances which emit flammable gases in contact with water, oxidizing substances; organic peroxides; toxic substances; infectious substances; radioactive material; corrosive substances and the last one miscellaneous dangerous substance [8-9]. Among the most common causes of accidents in the transport of dangerous substances are: natural catastrophes such as typhoon, floods, the great storm and landslides and human negligence according to a study carried out in Nigeria between 2007 and 2012 who demonstrated that $79 \%$ of the accidents were caused by man-made factors such as negligent and dangerous driving, resulting in $70 \%$ of cases of injuries and explosions that cause injuries, death or both $[7,10]$. 


\subsection{Typical robot for rescue operations and intervention in case of industrial hazard}

\subsubsection{Snake form robot}

The SnakeFighter Anna Konda presented in figure 2 is a robot designed to use in firefighting hazard operation that can identify and push the debris obstacle and based on the nozzle mounted in front part is able to extinguish fire. It uses the advantage of water pressure to move in space based on a hydraulic joint's construction combined to performance to resist under high temperature and under the cooling effect of water and it can be equipped with sensors and tools in accordance with rescue operation required by the hazard event, such as: video camera; temperature, pressure gas, noise/vibration sensors or welding tools etc. [11-12].

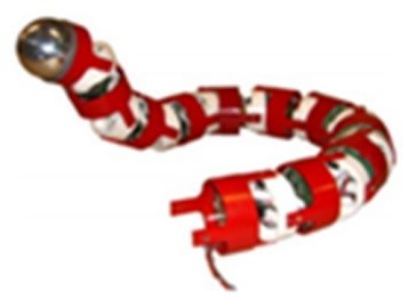

Fig. 1. SnakeFighter Anna Konda Robot

\subsubsection{Crawler-type robot}

The Servosila "Engineer" is a crawler type robot that can be used for rescue operations for anthropogenic hazard accident from industry as firefighting. This robot is equipped with tracks and it can be used to climb stairs, to traverse doorways and even narrow passage and also it offers the advantages to include sensors with radiation-hardened protection such as: GPS receiver, 3D laser scanner, stereo vision based on two cameras, one for rear-view and one for optical zoom. It also being equipped with a gripper to push and pull potentially dangerous object [13].

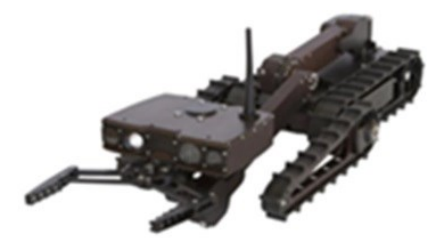

Fig. 2. The Servosila "Engineer" crawler-type robot

\section{Proposed solution}

The solution developed in this paper for emergency rescue intervention in case of industrial hazard accident is based on a Parrot mini-drone equipped with a network of sensors and a video camera capable of transmitting field data over post-explosion conditions to the control center for rapid identification of victims both in open and closed spaces. We also developed a mathematical model of the quadcopter drone along with the simulation of the control system and the image identification unit. 


\subsection{Hardware Configuration of Sensor Network}

In order to be able to make a decision in real time over the rescue operation type of potential victims based on the terrain data we choose to equipped our Parrot minidrone with CC2650 SensorTag development kit manufactured by Texas instruments presented in figure 3 .

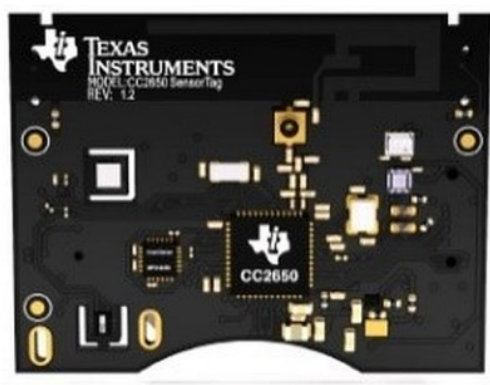

Fig. 3. The CC2650 SensorTag development kit

This is based on a powerful ARM® ${ }^{\circledR}$ Cortex ${ }^{\circledR}-\mathrm{M} 3$ CC2650 wireless microcontroller perfectly integrated that offering support for various communication protocol such as: Bluetooth Smart, ZigBee ${ }^{\circledR}$ or IPv6 protocol for personal network with low power consumption known as 6LoWPAN protocol in order to give the user a complete experience at very low power consumption [14].

The CC2650 SensorTag represent a useful solution in the environmental parameter acquisition due to an perfect integration along with a minidrone based on a series of key feature that it possess, such as: compact design $(5 \times 6.7 \times 1.4 \mathrm{~cm})$ with a low weight, only nine grams due to the fact this kit offers low power consumption, only a coin type battery (CR2032) offers 24 hours of functioning lifetime with minimal battery replacement [15]; it is expandable sensor - based device that incorporates SimpleLink TM Bluetooth Smart ${ }^{\circledR}$ technology offers a power consumption of less than $75 \%$ compared to previous series due to the implementation of the Bluetooth controller that is based on the iBeacon ${ }^{\mathrm{TM}}$ protocol having an architecture based on the standard IEEE 802.15.4 MAC protocol that gives a reduced code size [16]; offers remote access in cloud through Javascript programming language along with the Message Queue Telemetry Transport (MQTT) protocol so it can be accessed on both mobile applications and web pages; It is able to measure key environment parameters based on a network of ten MEMS sensors (micro-electro-mechanical systems) as follows: light ambiental sensor, infrared thermopile temperature sensor [17], thermopile ambiental sensor, accelerometer, gyroscope and magnetometer, air pressure sensor, air humidity sensor, microphone and magnetic sensor [18].

One of the most important sensors is infrared thermophile sensor that can detect temperature of the object without of necessity of direct contact based on infrared absorption energy emitted by object from the side of sensor view in a range between: $-40^{\circ} \mathrm{C}$ to $125^{\circ} \mathrm{C}$. However, even if the measured temperature range does not correspond to the ignition range for a potential fuel such as gasoline vapors $\left(415-530^{\circ} \mathrm{C}\right)$, hydrogen $\left(400^{\circ} \mathrm{C}\right)$ or methane $\left(538^{\circ} \mathrm{C}\right)$ based on thermopile in infrared, post-explosion outbreaks can be identified by combining the data provided by multiple sensors. In case of gas accumulation, the studies in domain demonstrated the fact that the methane represent a trigger for falling atmospheric pressure [19]. Thus, the data provided by the atmospheric pressure sensor can be just as important for managing the hazard situation as the data provided by the ambient light sensor that can be useful in accurately detecting the fire zones along with the images provided by the Parrot mini-drone camera [20]. 


\subsection{Physical solution based on mathematical modelation and simulation}

The quadcopter Parrot mini-drone that we use provides 6 degree of freedom ( 3 degree of freedom is reserved for translational movements and other three for rotational movements) that are obtained by coupling the rotation and translation movements.

We apply the direction cosine matrix to determine quadrotor movements equation from the ground to a fixed point:

$$
R_{Z X Y}=\left[\begin{array}{ccc}
C_{\Phi} C_{\theta} & C_{\phi} S_{\theta} S_{\Psi}-S_{\Phi} C_{\Psi} & C_{\Phi} S_{\theta} C_{\Psi}+S_{\Phi} S_{\Psi} \\
C_{\Phi} S_{\theta} & S_{\Phi} S_{\theta} S_{\Psi}+C_{\Phi} C_{\Psi} & S_{\Phi} S_{\theta} C_{\Psi}-C_{\Phi} S_{\Psi} \\
-S_{\theta} & C_{\theta} S_{\Psi} & C_{\theta} C_{\Psi}
\end{array}\right] \#(1)
$$

where:

$$
\begin{aligned}
& S_{\phi}=\operatorname{Sin}(\phi), C_{\Psi}=\operatorname{Cos}(\Psi), \text { etc.; } \\
& R \text { - matrix transformation; } \\
& \Phi \text { - Roll angle; } \\
& \theta \text { - Pitch angle; } \\
& \Psi \text { - Yaw angle. }
\end{aligned}
$$

Based on Lagrange approach we can determinated the dynamic model of the drone. Using the force and moment balance theory we can write the equations of motion as follows:

where:

$$
\left.\begin{array}{c}
\ddot{x}=u_{1}(\cos \Phi \sin \theta \cos \Psi+\sin \Phi \sin \Psi)-\frac{K_{1} \dot{x}}{m} \\
\ddot{y}=(\sin \Phi \sin \Psi \cos \Psi+\cos \Phi \sin \Psi)-\frac{K_{2} \dot{y}}{m} \\
\ddot{z}=u_{1}(\cos \Phi \cos \Psi)-g-\frac{K_{3} \dot{z}}{m}
\end{array}\right\} \#(2)
$$

$x$ - represent the forward position related to the axes of the earth;

$y$ - represent lateral position related to the axes of the earth;

$Z$ - represent vertical position related to the axes of the earth;

$K_{i}$ - represent the drag coefficients of the four motors.

In the following, based on the fact that the drag force is insignificant at a low speed and considering that the center of gravity should be at the intersection of the axes between the four motors we assume that stability increases as angular acceleration becomes less susceptible to forces once the center of gravity moves up or down. Another way to increase the stability can be realized by targeting of rotor forces to the center of the quadrotor body frame. This will reduce the effects of pitch and roll and also the vertical thrust. For convenience, we will define the inputs to be:

$$
\left.\begin{array}{c}
U_{1}=\left(T h_{1}+T h_{2}+T h_{3}+T h_{4}\right) / m \\
U_{2}=l\left(-T h_{1}-T h_{2}+T h_{3}+T h_{4}\right) / I_{1} \\
U_{3}=l\left(-T h_{1}+T h_{2}+T h_{3}-T h_{4}\right) / I_{2} \\
U_{4}=C\left(T h_{1}+T h_{2}+T h_{3}+T h_{4}\right) / I_{2}
\end{array}\right\}
$$

where:

$U_{1}$ - correspond to vertical thrust developed by the 4 rotors;

$U_{2}$ - represent the pitching effect;

$U_{3}$ - represent the yawing moment;

$U_{4}$ - represent the Rolling effect;

$T h_{i}$ - represent the thrusts force developed by four rotors; 
$I_{i}$ - represent the inertia intervals in relation to the axes.

In this paper we also developed a simulation in real time of Parrot Rolling Spider quadcopter behavior in development environment Matlab-Simulink based on some estimation and control modules that we found in a Matlab toolbox designed by the Massachusetts Institute of Technology (MIT). Based on designed estimation and control algorithms, Matlab-Simulink can generate automatically an embedded C-code that can be load onto the drone for test it's behavior in real-life.

In next figure is presented the Matlab Simulink model of drone's dynamics, sensor system and compensator.

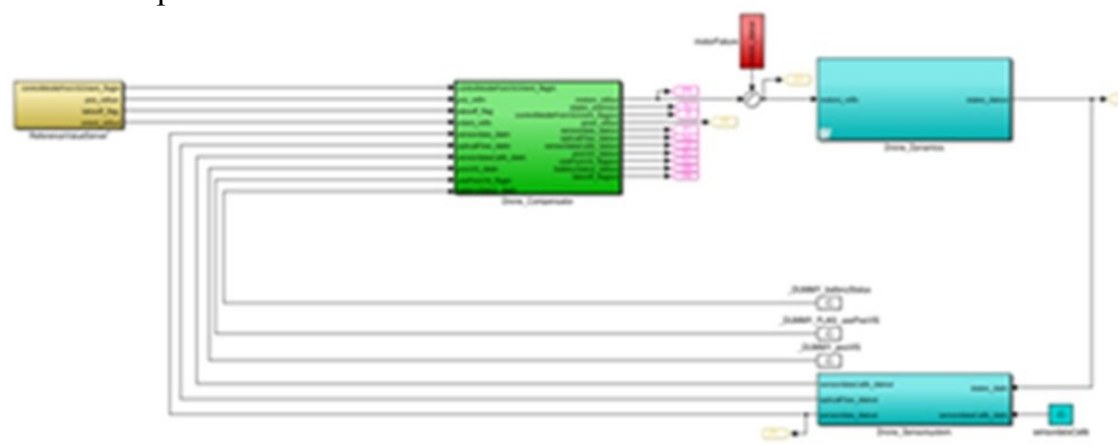

Fig. 4. Matlab Simulink model of drone Simulink model of drone's dynamics, sensor system and compensator

The plot resulted from simulated flight is presented in figure 5 and figure 6 .

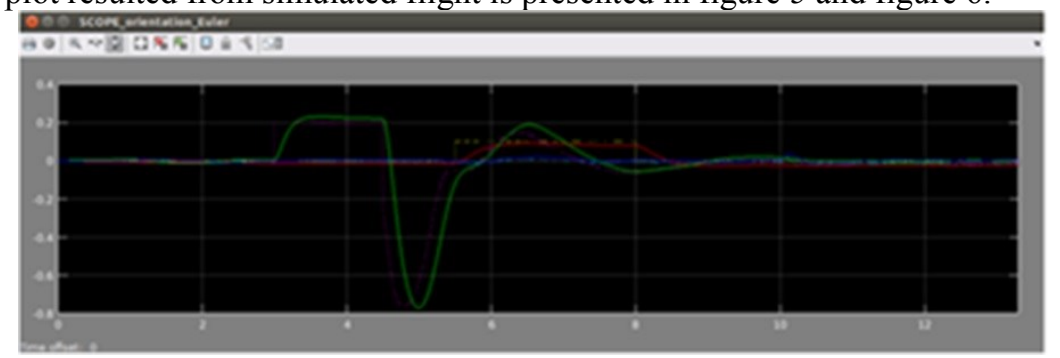

Fig. 5. Euler orientation simulation result

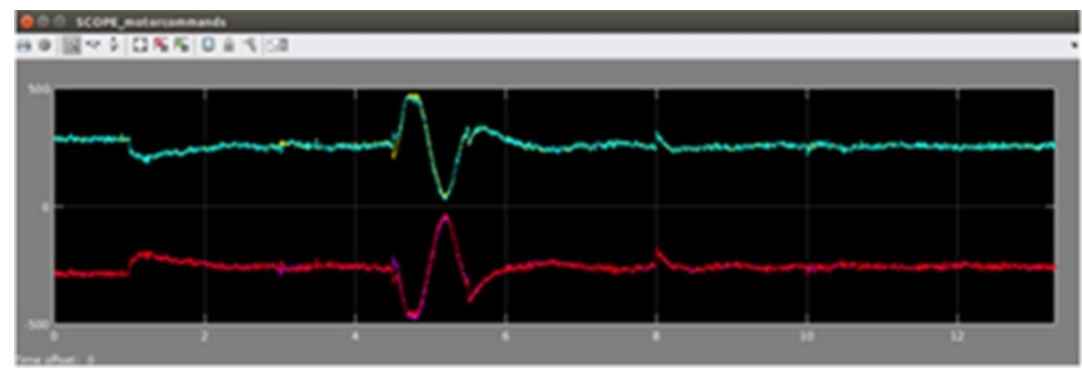

Fig. 6. Motor commands simulation result

\section{Conclusions}

The biggest problem in post-explosion rescue operations in industrial environments is the reaction time needed to gather information about the situation on the ground in order to 
decide how to intervene so as not to jeopardize the state of health of rescuers. For this reason, we offer a fast response solution for rescue operation on both open and closed spaces based on a mini-drone Parrot equipped with a low energy and lightweight sensor network through the CC2650 SensorTag development kit that provides a large number of environmental parameters from incident sites that can be accessed in the cloud such as: infrared object temperature together with visible ambient light to detect potential fire or potential victims from the affected area together with potential risk presented by the presence of combustible substances that can be detected by variations of atmospheric pressure and that can reactivate the hazard situation. In comparison to the solution offered by classical robots for rescue operations and interventions on the market such as snake foams or robots with track systems, the solution proposed by us based on drones offers the advantage of indirect interaction with the danger zone, as the mini-drone can cross the area affected by a safe altitude also offers the advantage of covering a larger area in a much shorter time than ground robots can be controlled by the operator outside the danger area based on real-time video streaming. The control system was modelled in Matlab-Simulink to develop the control system and for simulation functionality to improve the stability of the drone and to realize a direct control from the computer located at the command center and intervention.

\section{References}

1. R.M. Harrison, Pollution: causes, effects and control (Royal Society of Chemistry, 2015)

2. M. Risteiu, M. Leba, A. Arad, QUALITY - Access to Success 20, S1 341-346 (2019)

3. M.H. Beheshti, A.F. Chahak, A.A. Langari, S. Rostami,.Journal of Occupational Health and Epidemiology 4, 1-8 (2015)

4. L.N. Zheng, L. Zhang, L. Zhang, H.M. Liu, Advanced Materials Research 726-731, 1101-1104 (2013)

5. S. Rosca, S. Riurean, M. Leba, A. Ionica, Proceedings of the 2018 International Conference on Digital Science Springer, 235-242 (2018)

6. Z. Cimer, B. Szakal, Science for Population Protection 6, 1, 1-11 (2015)

7. W.H. Song, X.P. Bai, International Journal of Engineering 30, (8), 1144-1151 (2017)

8. Z. Yilmaz, E. Serpil, H.S. Aplak, Pamukkale University Journal of Engineering Sciences 22, 1, 39-53 (2016)

9. A. Ionica, M. Leba, R. Dovleac, Proceedings of the 12th Iberian Conference on Information Systems and Technologies 1882-1887 (CISTI) (2017)

10. H. Zhi-Qiang, Z. Ya-Mei, Procedia engineering 137, 843-848 (2016)

11. P. Liu, H. Yu, S. Cang, L.Vladareanu, Proceedings in the 2016 22nd International Conference on Automation and Computing (ICAC) IEEE,395-401 (2016)

12. P. Liljeback, O. Stavdahl, A. Beitnes, Proceedings in the 2006 9th International Conference on Control, Automation, Robotics and Vision IEEE, 1-6 (2006)

13. M. Sokolov, R. Lavrenov, A. Gabdullin, I. Afanasyev, E. Magid, Proceedings of the 4th International Conference on Control, Mechatronics and Automation 61-65 (2016)

14. Multi-standard CC2650 sensortag design guide (Texas Instruments Incorporated, 2015)

15. M. Mccarthy, P. Spachos, Proceedings of the 2016 IEEE 21st International Workshop on Computer Aided Modelling and Design of Communication Links and Networks (CAMAD) IEEE, 135-139 (2016)

16. T.T.T. Nguyen, Monitoring environmental variables using SensorTag (2017)

17. N. Patrascoiu, C. Rus, QUALITY - Access to Success 20, S1 347-352 (2019)

18. S. Riurean, S. Rosca, C. Rus, M. Leba, A. Ionica, Proceedings of the International Conference on Information Technology Science 47-55 (2017)

19. T. Tokida, T. Miyazaki, M. Mizoguchi, O. Nagata, F. Takakai, A. Kagemoto, R. Hatano, Global Biogeochemical Cycles 21, (2007)

20. O.M. Gamulescu, S.D. Rosca, M. Leba, A. Ionica, Proceedings of the 6th Review of Management and Economic Engineering International Management Conference 264-270 (2018) 